\title{
D oença arterial renovascular: seguimento dos pacientes apresentados como desafio terapêutico nas edições do Jornal Vascular Brasileiro 2003;2:35-6,85-7 e 2004;3:288-9,293-4
}

Arterial renovascular di sease: follow-up of the patients presented as therapeutic challenge in Jornal Vascular Brasileiro 2003;2:35-6,85-7 and 2004;3:288-9,293-4

\section{C asos clínicos}

\section{Caso clínico 1}

Paciente com hipertensão renovascular refratária ao tratamento clínico com crise hipertensiva e edema agudo de pulmão

Paciente feminina de 65 anos, interna na UTI com crise hipertensiva complicada por edema agudo de pulmão em junho de 1994. A paciente recebeu tratamento clínico, apresentou piora da função renal, sendo diagnosticadas trombose da artéria renal direita e estenose crítica da artéria renal esquerda. $\mathrm{N} \mathrm{a}$ época, foi indicado pelo autor o procedimento de revascularização renal esquerda para tratamento da hipertensão renovascular já descompensada. A paciente recebeu alta hospitalar pelo médico assistente, tendo sido considerada de risco proibitivo para a cirurgia pelo clínico. A paciente retornou 1 mês depois com o mesmo quadro, sendo internada para realizar o tratamento cirúrgico proposto. Foi real izada uma derivação da aorta torácica até a artéria renal esquerda via hiato diafragmático, com prótese de D acron, em agosto de 1994.

J Vasc Bras 2006;5(2):160-2

Copyright $\odot 2006$ by Sociedade Brasileira de Angiologia e de Cirurgia Vascular.
Evolução

A pacienteevoluiu com acreatinina variando de2,2 a 3,8 no pós-operatório e controle satisfatório da pressão arterial. A pacienteteverecuperação adequada, com pressão arterial controlada por um período de 10 anos (1994-2004). N o último ano, passou a apresentar perda progressiva da função renal. Entrou para o programa de hemodiálise em novembro de 2005. N o momento, encontra-se em programa de hemodiálise.

\section{Caso dínico 2}

Paciente com hipertensão arterial renovascular queevoluiu com anúria

Paciente feminina de 68 anos, foi internada com urgência apresentando edema agudo de pulmão e crise hipertensiva em maio de 2004. Foram diagnosticadas a trombose da artéria renal esquerda e a estenose crítica da artéria renal direita. 0 tratamento endovascular foi descartad o pelo radiologista na época. 0 cirurgião vascular não foi consultado. Recebeu alta com diagnóstico de insuficiência renal compensada e com indicação de tratamento dialítico. R etornou 20 dias após com nova crise de edema agudo de pulmão associada à anúria. Foi diagnosticada trombose da artéria renal direita, sendo então proposta e realizada, de imediato, a endarterectomia da artéria renal direita com re-implante na aorta. A recuperação pós-operatória imediata foi excelente. 
Evolução

A paciente permaneceu por 14 meses fora do programa de hemodiálise, com tratamento clínico para insuficiência renal, creatinina estável $(4,3)$, pressão arterial controlada e assintomática. Em dezembro de 2005, a pacienteretornou à emergência com quadro de piora da função renal, hipertensão, oligúria e dor lombar direita. Foi diagnosticada insuficiência renal crônica agudizada por trombose da artéria renal direita e iniciadaahemodiálise. N o momento, a pacienteencontra-se em programa de hemodiálise e clinicamente controlada.

\section{Comentários}

O s dois casos apresentados são exemplos de como intervenções cirúrgicas com técnica adequada podem atenuar a hipertensão renovascular, remover o risco do edema agudo de pulmão, evitar o óbito iminente e postergar a entrada do pacienteem programa de hemodiálise. O utro aspecto importante é a melhoria da qualidade de vida destas pessoas e a queda do custo de seu tratamento com múltiplas drogas anti-hipertensivas. 0 s procedimentos realizados tiveram durabilidade variável e compatível com a dificuldade diagnóstica encontrada. D eve ser ressaltado quea cirurgia foi aceita somentetardiamentepel osclínicos, quehiperavaliaram o risco do procedimento cirúrgico e minimizaram 0 risco da história natural da doença, sem revascularização renal.

0 grande ensinamento dos casos compilados é a remoção dos pacientes do risco de morte iminente. Além disso, os pacientes foram beneficiados com um longo período detratamento anti-hipertensivo de baixa dosagem, menor número de medicações e ausência de complicações clínicas. Permanecer fora do programa de hemodiálise propiciou um incremento na auto-estima e no convívio familiar, devendo ser citados como grandes ben efícios a curto, médio e longo prazos.

0 ganho pessoal e social dos casos acima pode ser medido de várias formas: no relato dos pacientes, nos custos econômicos que seriam gerados pela hemodiálise, além do tempo despendido para o tratamento ( $\mathrm{T}$ abelas 1 e2), bem como suas complicações com a piora na qualidade de vida.

Ambas as pacientes citadas tiveram redução da posologia das drogas anti-hipertensivas utilizadas para controle da pressão arterial, bem como troca de medicamentos. $\mathrm{N}$ o primeiro caso clínico, a paciente utilizava hidralazina $100 \mathrm{mg} 6 / 6 \mathrm{~h}$ e clonidina 0,150 mg 6/6 h, passando a utilizar, após a cirurgia, enalapril $20 \mathrm{mg} / \mathrm{dia}$ e atensina 0,325 mg/dia. No segundo caso clínico, a paciente fazia uso de atenolol $200 \mathrm{mg} / \mathrm{dia}$, hidralazina $100 \mathrm{mg} / \mathrm{dia}$ e metildopa 1,5 g/dia, passando a utilizar, após o procedimento de revascularização renal, atenolol $100 \mathrm{mg} / \mathrm{dia}$, furosemida $80 \mathrm{mg} /$ dia e nifedipina $60 \mathrm{mg} / \mathrm{dia}$.

$\mathrm{N}$ a Tabela 1, vemos uma demonstração, por paciente, do período de tempo sem hemodiálise, do número de sessões evitadas graças ao sucesso cirúrgico e do custo do tratamento cirúrgico recebido. Os dados são estimados com base em pacientes portadores de insuficiência renal crônica e tratamento dialítico padrão. É importante observar que a sobrevida do pacientecom insuficiência renal crônicapodenão ser longa (sobrevida de $40 \%$ em 5 anos), como a da

T abela 1 - D emonstração do tempo e sessões de hemodiálise evitadas pelo procedimento cirúrgico e seu custo

\begin{tabular}{lcccc}
\hline Paciente & $\begin{array}{c}\text { Tempo } \\
\text { fora da } \\
\text { hemodiálise }\end{array}$ & $\begin{array}{c}\text { Sessões de } \\
\text { hemodiálise } \\
\text { necessárias } \\
\text { sem cirurgia* }\end{array}$ & $\begin{array}{c}\text { Sessões de } \\
\text { hemodiálise } \\
\text { realizadas com } \\
\text { cirurgia bem-sucedida }\end{array}$ & $\begin{array}{c}\text { Custo do } \\
\text { tratamento cirúrgico } \\
\text { (SIH/SUS) } \\
\text { (R \$) }\end{array}$ \\
\hline Paciente 1 & 136 meses & 1.632 sessões & N enhuma sessão & $997,86+929,01^{\dagger}$ \\
Paciente 2 & 14 meses & 168 sessões & 4 sessões & 997,86 \\
\hline
\end{tabular}

SIH/SUS = Sistema de Informações Hospitalares do Sistema Único de Saúde.

* Média de 3 sessões semanais (adaptado de Vieira \& Luconi ${ }^{1}$ ).

† Procedimentos de revascularização renal e revascularização axilofemoral. 
paciente 1. Procuramos, através dessedado, demonstrar o custo de um tratamento dialítico prolongado. 0 utra observação importante é a de que os valores citados para os procedimentos cirúrgicos são os valores da tabela padrão de procedimentos para pacientes internados do Sistema de I nformações H ospitalares do Sistema Ú nico de Saúde (SIH /SU S).

$\mathrm{N} \mathrm{a}$ T abela 2, observamos os custos aproximados referentes ao tratamento dialítico que seriam despendidos caso as pacientes não tivessem sido operadas com sucesso. 0 custo da sessão dehemodiáli iseestá deacordo com a tabela do SUS, ou seja, é igual a R\$ 107,74 (M inistério da Saúde, SU S, 2004). É importante observar novamente que o período de tempo calculado para a paciente 1 pode não ser representativo da sobrevida média do paciente portador de insuficiência renal crônica. Salientamos ainda que não estão incluídos custos referentes a medicações utilizadas (eritropoetina, por exemplo), complicações do tratamento dialítico, internações, realização de fístulas arteriovenosas, colocação decateteres para hemodiálise, custoscom transporte ou despesas pessoais.

Concluindo, seria interessante citar a observação dos professores de radiologia do Cornell U niversity $M$ edical C enter (D ivisão de Radiologia C ardiovascular elntervencionista, $\mathrm{N}$ ew York $\mathrm{H}$ ospital), D avid T rost e Thomas Sos: "N ós cremos que nenhum doente deveria ser tratado com hemodiálise sem que uma causa reversível tenha sido afastada, corrigida ou eliminada".

Tabela 2 - C Custos da hemodiálise

\begin{tabular}{lcc}
\hline Paciente & $\begin{array}{c}\text { N úmero de sessões } \\
\text { de hemodiálise } \\
\text { necessárias (média) }\end{array}$ & $\begin{array}{c}\text { C usto da } \\
\text { hemodiálise* } \\
\text { (R \$) }\end{array}$ \\
\hline Paciente 1 & M ensal: 12,6 & M ensal: $1.357,52$ \\
& Anual: 152 & Anual: $16.376,48$ \\
Taciente 2 & M otal: 1722 & T otal: $185.528,28$ \\
& $\begin{array}{c}\text { Anual: } 152 \\
\text { T otal: } 177\end{array}$ & $\begin{array}{c}\text { M ensal: } 1.357,52 \\
\text { Tnual: } 16.376,48\end{array}$ \\
& 1.899 & $204.598,26$ \\
T otal & &
\end{tabular}

* Custo de uma sessão.
Agradecemos a consideração do senhor editor ao publicar esta carta, pois cremos que somente conhecendo os resultados a médio e longo prazo e divulgando os mesmos teremos melhoria no padrão de atendimento dos pacientes.

\section{Telmo Bonamigo}

Professor adjunto, D isciplina de Cirurgia Vascular, Fundação Faculdade Federal de C iências M édicas de Porto Alegre (FFFCM PA), Porto Alegre, RS. Chefe, Serviço de Cirurgia Vascular, Irmandade Santa C asa de M isericórdia de Porto Alegre, Porto Alegre, RS.

E-mail: telmobonamigo@terra.com.br

\section{João Carlos G oldani}

Professor adjunto, Disciplina de N efrologia, FFFCM PA, Porto Alegre, RS. Chefe, Serviço deN efrologia, I rmandade SantaC asa de M isericórdia de Porto Alegre, Porto Alegre, RS.

\section{Eduardo Lichtenfels}

Residente de Cirurgia V ascular, Serviço de Angiologia e Cirurgia V ascular, I rmandade Santa C asa de M isericórdia de Porto Alegre, FFFCM PA, Porto Alegre, RS.

\section{R eferências}

1. Vieira JA, Luconi P. Terapia renal substitutiva: estudo do financiamento dadiáliseno Brasil. Braśl lia: Associação Brasileira dos C entros de D iálise e T ransplante (ABCD T); 2004.

\section{Bibliografia recomendada}

1. M CC ready RA, Daugherty $M E, N$ ighbert $E J, H$ yde $G L$, Freedman AM, Ernsr CB. Renal revascularization in patients with singlefunctioning ischemic kidney. J V asc Surg. 1987;6: 185-90.

2. Messina LM, Zelenock GB, Yao KA, Stanley JC. Renal revascularization for recurrent pulmonary edema in patients with poorly controlled hypertension and renal insufficiency: a distinct subgroup of patients with arteriosclerotic renal artery occlusivedisease. J V asc Surg. 1992;15:73-80; discussion 80-2.

3. Sos TA, Trost DW. Renal vascular disease as a cause of hypertension. Curr O pin N ephrol H ypertens. 1995;4:76-81. 